\title{
Generadores e impulsores del marketing boca a boca en un programa de educación superior
}

\section{Generators and drivers of word of mouth marketing in a higher education program}

DOI: http://dx.doi.org/10.17981/cultedusoc.12.1.2021.15

Recibido: 2 de junio de 2020 Aceptado: 5 de octubre de 2020 Publicado: 31 de Diciembre de 2020

Darío José Espinal-Ruiz

I.U. Escuela Nacional del Deporte. Santiago de Cali (Colombia)

dario.espinal@endeporte.edu.co

Natali Cruz-González

I.U. Escuela Nacional del Deporte. Santiago de Cali (Colombia)

natali.cruz@endeporte.edu.co

\section{Luis Enrique David-Tenorio}

I.U Escuela Nacional del Deporte. Santiago de Cali (Colombia) luis.david@endeporte.edu.co

Para citar este artículo:

Espinal-Ruiz, D., Cruz-Gonzáles, N. y David-Tenorio, L. (2021). Generadores e impulsores del marketing boca a boca en un programa de educación superior. Cultura, Educación y Sociedad, 12(1), 227-240. DOI: http://dx.doi.org/10.17981/cultedusoc.12.1.2021.15

\section{Resumen}

En la actualidad, el uso de medios tradicionales de promoción y marketing no ha resultado ser muy efectivo para las instituciones de educación superior por cuanto no se evidencian los efectos esperados. Consecuentemente, el presente artículo tiene como objetivo identificar los generadores e impulsores del marketing boca a boca a boca en un programa de educación superior. Para la realización de la investigación se empleó una metodología cuantitativa, a través de un muestreo probabilístico estratificado donde se aplicaron 185 encuestas a estudiantes del programa de Administración de Empresas de la Institución Universitaria Escuela Nacional del Deporte (Cali, Colombia). Los resultados obtenidos muestran que los estudiantes son los principales generadores del boca a boca, y que el mayor impulsor radica en el aspecto diferenciador del programa académico y la reputación de la institución.

Palabras clave: Gestión educativa; comunicación; marketing educativo; educación superior

\section{Abstract}

At present, the use of traditional means of promotion and marketing has not turned out to be very effective for higher education institutions because the expected effects are not evident. Consequently, this article aims to identify the generators and drivers of word of mouth marketing in a higher education program. To carry out the research, a quantitative methodology was used, through stratified probabilistic sampling where 185 surveys were applied to students of the Business Administration program of the National School of Sports University Institution (Cali, Colombia). The results obtained show that students are the main generators of word of mouth, and that the greatest driver lies in the differentiating aspect of the academic program and the reputation of the institution.

Keywords: Education management; communication; educational marketing; higher education 


\section{INTRODUCCIÓN}

La interacción de las organizaciones con su comunidad de seguidores es una prioridad (Godin, 2009). La necesidad del individuo de estar conectado con otros para compartir intereses comunes, convierte al boca a boca en un importante medio de marketing educativo. El boca a boca se produce cuando los usuarios interactúan y muestran la efectividad de su opinión o recomendación a otras personas (Stauss, 2000). Aunque se reconozca su gran utilidad, diversos autores solo mencionan sus beneficios y no amplían sobre su utilización y potenciación (Wolny \& Mueller, 2013), especialmente en el contexto universitario en el cual resulta relevante conocer sus impulsores y generadores, de manera que, se puedan orientar los procesos de mercadeo educativo eficientemente (Herold, 2015), siendo una necesidad latente para toda institución de educación superior tomando en cuenta las altas tasas de deserción de estudiantes (Olarte, 2020).

La comunicación de boca a boca es una de las más antiguas formas de recomendar o intercambiar opiniones sobre un producto o servicio, este tipo de difusión se realiza cuando alguien muestra su opinión positiva o negativa y se lo transmite a otra persona generando una cadena de comunicación de largo alcance (Park \& Kim, 2014), la cual influye sobre consumidores futuros (Filieri, 2015). El boca a boca es una forma de transmisión oral de un mensaje, en la que el receptor no percibe el mensaje como publicidad de un producto o servicio (Aguilar, San Martín y Payo, 2014). Aquí radica el éxito del marketing boca a boca: la persona que recibe el mensaje no estará predispuesta a publicidad o similares, facilitando la aceptación del mismo. Este funciona a través de la transmisión de información sobre productos o servicios de una persona a otra (Solomon, 2013), que puede llegar a convertir a desconocidos en parte de una comunidad con intereses compartidos (Kozinets, de Valck, Wojnicki \& Wilner, 2010), quienes consideran más confiable este tipo de información con relación a la suministrada por otro medio (Bronner \& Hoog, 2010; Sham \& Nasr, 2016), la cual pasa a convertirse en el fundamento que determina el análisis en la selección final de consumo (Gupta \& Harris, 2010).

A nivel educativo el marketing boca a boca define un esfuerzo organizado y liderado por una institución de educación que tiene la intención de convencer y comprometer a su comunidad educativa con una serie de ideas, actitudes, prácticas y comportamientos las cuales representen la filosofía y los valores organizacionales a transmitir a la sociedad (Cihovska, 2013). Esto representa una función indispensable de las instituciones educativas en el entorno competitivo de hoy en día, ya que resulta fundamental proyectar una imagen que genere valor para sus distintos stakeholders y permita atraer una mayor cantidad de talento humano y recursos financieros (Stachowski, 2011) para asumir los retos establecidos por la agenda del desarrollo sostenible y la emergencia de la Cuarta Revolución Industrial (Espinal, Scarpetta y Cruz, 2020).

A pesar de su importancia, existe una brecha en la literatura que permita comprender los impulsores y generadores del boca a boca como estrategia de promoción de servicios educativos en instituciones universitarias (Bhayani, 2015). El desconocimiento de los generadores e impulsores del boca a boca puede limitar el diseño e implementación de una estrategia de marketing educativo que estimule en la comunidad educativa la intención positiva de recomendación a otros potenciales estudiantes (Chen, 2016). De acuerdo con este contexto, 
el presente artículo tiene como objetivo identificar los generadores e impulsores del boca a boca a boca para un programa de educación superior, de acuerdo al caso de estudio del programa académico de Administración de Empresas de la Institución Universitaria Escuela Nacional del Deporte (Cali, Colombia).

Las implicaciones prácticas de este articulo residen en brindar herramientas para la optimización de los procesos de mercadeo educativo en instituciones de educación superior que pretenden generar espacios alternativos de promoción de sus servicios educativos de manera eficiente. El presente artículo se estructura en las siguientes partes. En la introducción se define el problema de investigación y el contexto general del estudio. Seguido, en la revisión de la literatura se analizan los fundamentos teóricos del boca a boca como estrategia de marketing educativo. En la metodología, se expone el diseño adoptado para este estudio. Y finalmente, se presentan los resultados de investigación, la discusión y las conclusiones del estudio.

\section{REVISIÓN DE LA LITERATURA}

Una visión favorable por parte del consumidor acerca de un producto o servicio facilita el boca a boca positivo, generando así opiniones optimistas, de lo contrario, perjudicará las decisiones de compra (Kim \& Johnson, 2016). Por lo anterior, Eisingerich, Chun, Liu, Jia \& Bel (2015) manifiestan que la conversación entre los individuos se basará en las experiencias vividas formando opiniones informales como reflejo de la percepción acerca de la imagen de una marca determinada. Los consumidores suelen buscar referencias en otras personas dentro de un círculo cercano, lo cual les ofrece más confianza en el mensaje con respecto a otro medio de comunicación (Reinares \& Reinares, 2007). Es así como en la actualidad los consumidores tienen al boca a boca como una fuente de información más creíble e influyente que la proveniente de fuentes comerciales (Kotler, Kartajaya \& Setiawan, 2010; López \& Sicilia, 2013).

El boca a boca es vital para las organizaciones que buscan tener un mayor éxito en sus objetivos de marketing frente a sus similares que no lo aprovechan (Jaakkola \& Aarikka, 2018; Mazzarol, Sweeney \& Soutar, 2007). Desde el punto de vista de las instituciones educativas, el intercambio relacional con la comunidad educativa contribuye a la diferenciación del servicio educativo proporcionando una ventaja competitiva (Farrel, 2001). En este contexto, diversos estudios han demostrado la efectividad del boca a boca como parte de la estrategia de marketing educativo en instituciones de educación superior (Le, Dobele \& Robinson, 2019; Ansari, Hurriyati, Gaffar, Adi \& Amanah, 2017). El primer desafío consiste en presentar y explicar adecuadamente el servicio educativo a su comunidad como parte del proceso de atraer una masa crítica de talento humano de clase mundial (Salmi, 2009). En esta fase, un canal de comunicación importante es el boca a boca, el cual se origina al interior de las instituciones como resultado de la experiencia de egresados, padres de familia, profesores, personal administrativo, empleadores y otros estudiantes, además de las opiniones disponibles por otros medios de comunicación (Enache, 2011).

Para algunos autores, los medios tradicionales tienden a ser poco efectivos (Olle y Riu, 2010). El boca a boca puede ser la respuesta a cómo lograr que el mensaje del servicio educativo llegue al consumidor y este realmente lo quiera escuchar o aceptar (Sernovitz, 
Godin y Kawasaki, 2009; Libai et al., 2010). Es importante precisar que, aunque esta estrategia es un medio para darse a conocer, es también fundamental la credibilidad de quien lo genera. En ese momento la persona que recibe la recomendación produce la evaluación de esta a partir del conocimiento que la persona tenga de su valor (Soares, Lapuente y De matos, 2015). La aceptación del mensaje depende en muchas ocasiones de la seguridad del canal de transmisión, por tanto, la apropiación del mensaje será más profunda si las opiniones provienen de personas en las que se deposita alguna confianza, tales como amigos y familiares (Basso, Reck y Rech 2013; Scheers \& Prinsloo, 2014). Las interacciones entre consumidores tanto fuera de línea como en línea constituyen los medios más efectivos para la transmisión del boca a boca, siendo facilitadores para la comunicación del mensaje (Karlíček, Tomek \& Kř́ízek, 2010).

El marketing boca a boca se ha consolidado como una herramienta de transmisión de mensajes hacia los grupos meta de alta efectividad. Trusoy, Buclin y Pauwels (2009) muestran como esta estrategia es una gran oportunidad para la promoción, a partir de un pequeño grupo de promotores que comparten información confiable se puede generar un mensaje viral. Es así como se hace necesario la generación del compromiso del cliente, el cual se debe basar en valores e identificación con la organización, generando un efecto positivo en la lealtad de este, conllevando al impulso y generación del boca a boca (Fullerton, 2003). Aun cuando el boca a boca es un tema bastante estudiado, se evidencia un vacío en la literatura que explique los generadores e impulsores en instituciones educativas, específicamente en la oferta de servicios educativos, tales como programas académicos de educación superior (Lehmann, 2017). Por tal razón, esta investigación pretende aportar en la disminución de esta brecha del conocimiento evaluando un caso práctico del uso del boca a boca en el programa académico de administración de empresas de la Institución Universitaria Escuela Nacional del Deporte (Cali, Colombia).

\section{Metodología}

Utilizando un enfoque cuantitativo de tipo descriptivo y relacional se analizaron las distintas estrategias de boca a boca para la promoción de servicios educativos en una institución de educación superior. Como unidad de análisis principal se tomó el programa de Administración de Empresas de la Institución Universitaria Escuela Nacional del Deporte, organización de carácter público adscrita al Ministerio del Deporte y al municipio de Santiago de Cali (Colombia).

El proceso investigativo se dividió en dos fases. En la primera fase, se diseñó y validó un cuestionario estructurado en cinco variables de análisis para la recolección de los datos (Tabla 1). Se adaptaron dos variables que miden las intenciones del boca a boca (generadores-impulsores) (Fullerton, 2003), las cuales fueron evaluadas de dos maneras, una descriptiva en una escala nominal y otra de manera inferencial en una escala ordinal. También se adaptaron dos variables de evaluación de las estrategias de mercadeo educativo, y los medios de divulgación según la propuesta de Karlíček et al. (2010); y se construyó una categoría que evalúa los datos de caracterización general de la población. En la segunda fase, para la selección de la muestra se realizó un muestreo probabilístico estratificado dividido en 12 estratos (siete semestres diurnos y cinco semestres nocturnos). 
El cálculo del tamaño de la muestra se realizó con un margen de error máximo del 4,5\% y un nivel de confianza del 97\%. En total, se encuestaron 185 estudiantes, que correspondían al 68\% de la población con la cual contaba el programa de Administración de Empresas al momento de realizar el estudio.

El procesamiento de datos se realizó utilizando el software SPSS (versión 26) mediante el módulo de muestras complejas. Se realizó un análisis estadístico descriptivo univariado para las cinco variables propuestas, y se aplicó una regresión lineal simple entre las variables de generadores e impulsores del boca a boca.

TABLA 1.

Variables de análisis

\begin{tabular}{lll}
\hline \multicolumn{1}{c}{ Variables } & \multicolumn{1}{c}{ Definición } & \multicolumn{1}{c}{ Fuente } \\
\hline Caracterización de la población. & Género; Edad; Jornada de Estudio. & Elaboración propia. \\
Generadores boca a boca. & $\begin{array}{l}\text { Personas que recomiendan el programa; } \\
\text { Compromiso afectivo. }\end{array}$ & Fullerton (2003). \\
Impulsores boca a boca. & $\begin{array}{l}\text { Razones para recomendar el programa; } \\
\text { Apoyo al programa. }\end{array}$ & Fullerton (2003). \\
Medios de divulgación. & $\begin{array}{l}\text { Medios usados por el programa para su } \\
\text { promoción. }\end{array}$ & Karlíček et al. (2010). \\
Estrategias mercadeo educativo. & Estrategias del programa y su impacto. & Karlíček et al. (2010). \\
\hline
\end{tabular}

Fuente: Adaptado a partir de Fullerton (2003) y Karlíček et al. (2010).

\section{Resultados}

Los resultados de investigación incluyen la caracterización de la población, los generadores, impulsores, medios de comunicación y la relación que existe entre los generadores e impulsores del boca a boca para un programa académico de educación superior. Los resultados a nivel descriptivo indican que para la población joven menor de 20 años los mayores generadores del boca a boca son los estudiantes de la misma institución los cuales impulsan el aspecto diferenciador del programa como su principal atributo. A nivel, relacional los resultados indican que los atributos distintivos del programa influyen significativamente sobre la intención de recomendar a otros potenciales estudiantes.

\section{Caracterización de la población}

De la muestra seleccionada, el $40 \%$ pertenecen al género femenino y el $60 \%$ al género masculino. Respecto a la edad, el 23\% se encuentran en el rango de edad de los 15 a 18 años; el 37\% de los 19 a 20 años; el 28\% de 21 a 25 años; el 6\% de 25 a 30 años y el 6\% superior a 30 años. En cuanto a la participación por jornadas de estudio, el 51\% de la muestra pertenecía a la jornada diurna y el $49 \%$ a la nocturna. En general, se trata de una población joven, cuya mayor concentración se encuentra en los rangos inferiores a 20 años. 


\section{Generadores del boca a boca}

Los generadores del boca a boca son quienes realmente hablan del programa. En este caso quien genera la conversación. Los resultados muestran que los estudiantes de otros programas académicos de la institución (39\%) y del programa de Administración de Empresas (35\%) son los mayores generadores de opinión y recomendación del programa; seguido de empleados de la institución (12\%), docentes (10\%) y empleadores (4\%). Adicional a identificar cuáles eran los generadores del boca a boca, se esperaba conocer qué vínculo tenían con el mismo. En este caso, los mayores influyentes en la comunicación del mensaje son los amigos (53\%), la familia (29\%), los compañeros de entrenamiento (10\%) y de trabajo (8\%). De esta manera, se corrobora que el boca a boca parte desde la confianza, un amigo o un familiar solo recomienda lo que genera efectos positivos a sus personas allegadas. Aparentemente, esto implica que la toma de decisiones en el momento de la compra del servicio educativo es influenciada por el comportamiento de las personas allegadas a los potenciales estudiantes.

\section{Impulsores del boca a boca}

Para identificar los impulsores del boca a boca entre sus generadores, se trabajó sobre los aspectos diferenciadores del programa reconocidos por el estudiante en el momento de optar por el mismo. Elementos como los horarios ofertados por el programa (17\%) y las instalaciones deportivas (17\%) son algunos de los temas identificados. Sin embargo, en mayor proporción (27\%) los estudiantes identifican que la proyección del programa por su aspecto diferenciador hace énfasis en la gestión deportiva como motivador más importante. Al tener en cuenta los anteriores resultados, se deduce que el punto de partida para la promoción del programa se relaciona a aspectos intangibles fundamentados en los componentes académicos que lo distinguen de otros programas del mismo tipo. Por lo anterior, impulsar el boca a boca de una manera orgánica genera mayor impacto a través del uso de aspectos tangibles como incentivos o en otro tipo de recursos que el potencial estudiante puede percibir como beneficiosos.

\section{Medios de comunicación}

La oferta de medios del programa está representada entre presencia en la web, medios tradicionales, visitas a colegios y el boca a boca. La transmisión del boca a boca ocupa el $48 \%$ de preferencia, siendo el medio más popular de divulgación como fuente de información creíble e influyente en la toma de decisiones para inscribirse en el programa. Los otros medios de comunicación presentan los siguientes resultados: la página web con un $22 \%$, las visitas a colegios con el 19\%, y con menor participación, los medios tradicionales con solo el 11\%. El resultado evidencia que el uso de medios tradicionales de promoción no resulta tan efectivo y su impacto es mínimo. Es aquí donde radica el éxito del marketing boca a boca, la persona que recibe el mensaje no está predispuesta a publicidad o similares, facilitando la aceptación de este. En este aspecto recae la importancia de transmitir el diferenciador institucional y del programa, que impulsa el boca a boca entre los miembros de la comunidad impactada. 


\section{Estrategias del boca a boca}

Los resultados obtenidos evidencian que los comentarios a través de las redes sociales de parte de los estudiantes son positivos. Sin embargo, el contenido generado no se puede correlacionar con el aumento en el nivel de las inscripciones al programa, por cuanto la comunidad que sigue las diferentes redes son en su mayoría estudiantes activos y egresados, por tal motivo, las redes institucionales y del programa no son un predictor confiable. Así mismo, los medios tradicionales como radio, prensa y televisión no han resultado efectivos. Esto se pudo evidenciar comparando los niveles de inscripción de estudiantes nuevos frente a periodos que se hicieron campañas publicitarias y periodos donde no se hicieron. Comparando estos datos, no se evidencian diferencias significativas en los niveles de matrículas. Consecuentemente, se deduce que no han sido estrategias efectivas. Aunque la única estrategia formal de boca a boca entre las comunidades es la utilización de campañas de referidos, estas no han resultado efectivas, se observa que el $32 \%$ de los estudiantes manifiesta no tener referidos o a quien recomendar el programa y el $12 \%$ desconocer las campañas.

De acuerdo con esto, la palabra referido implica que el estudiante deba realizar la acción de vender el programa o hablar del mismo en pro del incentivo económico. Como consecuencia, los resultados de investigación indican que impulsar el boca a boca de una manera orgánica genera mayor impacto que a través del uso de incentivos económicos, por cuanto, cuando se indaga en la comunidad de estudiantes nuevos sobre su decisión de matrícula, el 48\% afirma que se debe a la reputación del programa, ya sea, por recomendación de familiares y amigos. En este sentido, se puede afirmar que el impacto de la estrategia del boca a boca se origina informalmente, no obstante, ha tenido mejores resultados frente a las estrategias formales.

\section{Influencia de los impulsores sobre los generadores del boca a boca}

Para identificar la influencia que tienen los aspectos diferenciadores del programa sobre la intención que tienen las personas para recomendar el programa, se realizó una regresión lineal simple, donde se tomó como variable dependiente los generadores del boca a boca y como variable independiente los impulsores (Tabla 2).

TABla 2.

Estadísticas de la regresión lineal simple

\begin{tabular}{ll}
\hline \multicolumn{1}{c}{ Estadísticas } & Resultados \\
\hline Coeficiente de correlación & 0,83 \\
Coeficiente de determinación $\mathrm{R}^{2}$ & 0,69 \\
$\mathrm{R}^{2}$ ajustado & 0,65 \\
Estadístico t & 4,22 \\
Valor critico de $\mathrm{F}(\mathrm{p})$ & 0,002 \\
\hline
\end{tabular}

Fuente: Elaboración propia a partir de SPSS (v. 26). 
El resultado de la prueba indica que los aspectos diferenciadores del programa influyen significativamente sobre la intención de recomendar a otras personas que se vinculen al mismo $(p=0,002)$. La alta correlación demostrada por las variables, corrobora que los aspectos diferenciadores aparentemente tienen una relación con la decisión de los estudiantes para recomendar a otras personas vincularse al programa. El resultado del $\mathrm{R}^{2}$, muestra que los impulsores influencian en un 69\% la decisión de recomendación de los estudiantes, esto implica que el otro $31 \%$ puede ser explicado por otras variables no tenidas en cuenta en la prueba, sin embargo, es un valor predictivo aceptable.

Los resultados de investigación demuestran que son los estudiantes de la institución los generadores del boca a boca, siendo el aspecto diferenciador del programa el factor que más impulsa la decisión de recomendación. En consecuencia, se puede afirmar que ha sido la comunidad educativa la encargada de promover la filosofía, valores y reputación institucional plasmada en el programa de Administración de Empresas el cual se encuentra posicionado en el contexto local por su énfasis en gestión deportiva.

\section{DisCUSIÓN}

Los resultados de la investigación corroboran que el boca a boca es la estrategia con mayor efectividad para dar a conocer el programa entre la comunidad; la confianza generada por la recomendación o el comentario de amigos y familiares resulta siendo un factor clave para atraer nuevo talento humano al programa académico. Esto se alinea con lo propuesto por Kotler et al. (2010), donde se afirma que la información proveniente de fuentes confiables es más efectiva frente a la proveniente de fuentes comerciales. No obstante, se podría afirmar que, para el caso de estudio en mención, el boca a boca emerge como una estrategia informal correspondiente en mayor medida a factores contingenciales y coyunturales provenientes de aspectos relacionados a la reputación, imagen y lealtad con respecto a la marca de la institución universitaria. Por tanto, para aumentar el impacto de esta estrategia, resulta relevante combinar el medio tradicional persona a persona, con estrategias de boca a boca con la utilización de medios digitales.

En lo relacionado a los generadores del boca a boca, los resultados muestran que los estudiantes son el principal medio de recomendación del programa. Aparentemente, esto se puede atribuir al intercambio relacional entre su comunidad educativa, otorgando de esta manera una ventaja competitiva frente a otras instituciones (Farrel, 2001). Adicional a esto, la comunicación del mensaje proviene principalmente de amigos y familiares, resultado que se alinea con lo propuesto por Trusoy et al. (2009), quienes sostienen que a partir de un pequeño de grupo de personas que comparten con sus contactos información confiable se puede generar un mensaje de un amplio alcance entre una comunidad que comparte intereses similares, que para el caso de estudio se relacionan a los intereses en la administración deportiva. En este sentido, tal como lo propone Enache (2011), el mensaje proviene del interior de la institución producto de la experiencia de los estudiantes, el cual es transmitido a familiares y amigos para luego reproducirse en los círculos de confianza de sus allegados. 
Cuando se trata de impulsar el boca a boca, Sernovitz et al. (2009) sugiere que se debe dar a las personas una razón para facilitar el desarrollo de conversaciones sobre el servicio. En la investigación se identificó que el impulsor principal del boca a boca en el programa era su énfasis en administración deportiva. Por tanto, la razón por la cual las personas hablan del programa se origina en su aspecto diferenciador. De acuerdo con esto, el diferenciador de un programa académico se convierte en un motivador de confianza e impulsador de su recomendación. Lo anterior es confirmado por Basso et al. (2013), cuando sostiene que la generación del boca a boca parte de la credibilidad y confianza sobre el servicio. Soares et al. (2015) comentan que no es solo compartir el mensaje, también se debe confiar en lo compartido. Entonces, se puede deducir que para atraer talento humano al programa es necesario fundamentar las campañas de comunicación sobre su aspecto diferenciador y proyección de futuro buscando que más personas hablen sobre el mismo y así producir la viralidad del mensaje.

En cuanto a las estrategias implementadas, los resultados demuestran que los medios de comunicación tradicionales han sido poco efectivos para la vinculación de nuevo talento humano al programa. Esto se corresponde con lo expuesto por Olle y Riu (2010) y Aguilar et al. (2014), quienes sostienen que los mensajes percibidos como publicidad de un producto o servicio tienen menor impacto sobre la intención de consumo. Por tanto, una estrategia de comunicaciones para servicios educativos se debe enfocar en la generación de valor social, en la credibilidad y los valores institucionales (Le et al., 2019). Así mismo, los medios digitales son tratados exclusivamente como medios informativos y no como difusores de las ideas, actitudes, prácticas y comportamientos que se quieren transmitir para comprometer a su comunidad educativa (Cihovska, 2013), por tanto, tienen un impacto reducido en los procesos de marketing educativo del programa. Finalmente, la estrategia formal de boca a boca por medio de referidos, no ha sido efectiva. Esto implica que los resultados en la atracción de talento humano han correspondido más a un crecimiento orgánico que a la disposición de incentivos económicos.

\section{Conclusiones}

Los principales generadores del boca a boca en el programa de Administración de Empresas son sus estudiantes. Este resultado afirma el postulado que la confianza con relación a quien transmite el mensaje es superior a la proveniente de los medios tradicionales. Por tanto, una buena experiencia del estudiante en la prestación del servicio educativo, es la mejor forma para impulsar la reproducción de un mensaje positivo acerca de los aspectos diferenciadores del programa, que, para este caso, es el énfasis en administración deportiva.

El boca a boca, en este caso, se generó sin el uso de redes sociales. Aunque el programa tiene presencia en la web por medio de la página de la Institución y Facebook, su impacto en la decisión final de los estudiantes para su inscripción en el programa es menor que la del boca a boca. Por tanto, la presencia en redes sociales y una buena gestión de contenidos que permita identificar impulsores y generadores, probablemente redunde en resultados positivos para el programa. Por otro lado, el uso 
de las campañas de referidos evidencia resultados ambiguos, para obtener resultados efectivos se requiere consolidar una estrategia interna de divulgación que permita a la comunidad estudiantil se apropie del mensaje para impulsarlo, no obstante, se evidencia que el estudiante no está dispuesto a asumir este rol, ello evidencia que el incentivo económico no es un disparador eficaz del boca a boca en el programa académico de Administración de Empresas.

Los hallazgos evidencian que la estrategia de marketing educativo se fundamenta en la comunicación de la reputación que ha consolidado el programa basado en su aspecto diferenciador. Sin embargo, no se puede afirmar que se ha implementado una estrategia formal, ya que los resultados demuestran que ha sido el boca a boca orgánico el principal impulsor de la reputación del programa. Por tanto, se puede deducir que al focalizar los esfuerzos en una estrategia formal se podría lograr una mejor eficiencia en el uso de los recursos y mayor eficacia en la cantidad de talento humano que se atrae. Al revisar la estrategia general de marketing educativo, el estudio demuestra la necesidad de un abordaje distinto al que se promueve en el marketing comercial, la comunicación de las ideas y valores de una institución educativa requieren mecanismos diferenciales para llegar al público objetivo por canales distintos a los tradicionales, esto implica que el tratamiento de la estrategia de marketing educativo, de acuerdo a las características del público objetivo de la institución, debe tener un énfasis en el ámbito social y deportivo.

\section{Limitaciones e investigación futura}

Aunque este estudio proporciona hallazgos significativos sobre los generadores e impulsores del boca a boca para programas de educación superior, persisten algunas limitaciones, lo que suministra las direcciones para la investigación futura. En primer lugar, las conclusiones de estudio se limitan al caso de estudio del programa de Administración de Empresas de la Institución Universitaria Escuela Nacional del Deporte en Colombia, restringiendo la generalización de los resultados a otros programas académicos e instituciones de educación superior. La investigación futura debería replicar este estudio en otras programas e instituciones que permitan la comparación en contextos culturales y económicos diversos.

En segundo lugar, este estudio es transversal, por lo tanto, no se considera cómo han evolucionado los resultados del boca a boca en el tiempo. La investigación futura debería considerar realizar un estudio longitudinal que permita comparar sus resultados en diferentes periodos de tiempo. En tercer lugar, aun cuando se consideran variables referenciadas en la literatura, es posible que algunos factores hayan sido omitidos. Por lo tanto, la investigación futura debería integrar otras variables como fundamento a un análisis con mayor impactos en una estrategia integral de marketing educativo. Finalmente, al ser un estudio de tipo descriptivo y relacional que utiliza el análisis univariado y la regresión lineal simple puede presentar limitaciones metodológicas en cuanto al alcance de los resultados, no obstante, se considera que el estudio se constituye como un buen punto de partida de un programa de investigación sobre la estrategia del boca a boca en instituciones de educación superior. 


\section{REFERENCIAS}

Aguilar, V., San Martin, S. \& Payo, R. (2014). La aplicación empresarial del marketing viral y el efecto boca-oreja electrónico. Cuadernos de Gestión 14(1), 15-31. https://doi. org/10.5295/cdg.120348va

Ansari, D., Hurriyati, R., Gaffar, V., Adi, L. \& Amanah, D. (2017). Effect of Word of Mouth on Students Decision to Choose Studies in College. In: SCITEPRESS, 1st International Conference on Islamic Economics, Business, and Philanthropy (pp. 793797), Bandung, Indonesia. https://doi.org/10.5220/0007090007930797

Basso, K., Reck, D. y Rech, E. (2013). Personalidad y boca la boca: propuesta al envio y recepción de información. Revista de Administración de Empresas, 53(6), 580-591. https://doi.org/10.1590/S0034-75902013005000004

Bhayani, A. (2015). Social and peer influences in college choise. In: World Academy of Science, Engineering and Technology, International Conference on Economics and Business Market Management (pp. 1-20), Trieste, Italy. Available: https://ro.uow.edu. au/dubaipapers/694/

Bronner, A. \& Hoog, R. (2010). Consumer-generated versus marketer-generated websites in consumer decision making. International journal of market research, 52(2), 231-248. https://doi.org/10.2501/S1470785309201193

Chen, C. (2016). The Investigation on Brand Image of University Education and Students' Word-of-Mouth Behavior. Higher Education Studies, 6(4), 23-33. https://doi. org/10.5539/hes.v6n4p23

Cihovska, V. (2013). Social Orientation of Marketing Concept. Journal of Positive Management, 4(1), 52-62. https://doi.org/10.12775/JPM.2013.004

Eisingerich, A., Chun, H., Liu, Y., Jia, H. \& Bell, S. (2015). Why recommend a brand face-to-face but not on Facebook? How word-of-mouth on online social sites differs from traditional word-of-mouth. Journal of Consumer Psychology, 25(1), 120-128. https://doi.org/10.1016/j.jcps.2014.05.004

Enache, I. (2011). Marketing higher education using the 7 ps framework. Bulletin of the Transilvania University of Braşov, 4(53), 23-30. Available: http://webbut.unitbv.ro/ Bulletin/

Espinal, D., Scarpetta, G. y Cruz, N. (2020). Análisis prospectivo estratégico de la educación superior en Colombia. Cultura, Educación y Sociedad, 11(1), 177-196. http:// dx.doi.org/10.17981/cultedusoc.10.2.2020.13

Farrel, F. (2001). Postmodernism and Educational Marketing. Educational Management \& Administration, 29(2), 169-179. https://doi.org/10.1177/0263211X010292003

Filieri, R. (2015). What makes online reviews helpful? A diagnosticity-adoption framework to explain informational and normative influences in e-WOM. Journal of Business Research, 68(6), 1261-1270. https://doi.org/10.1016/j.jbusres.2014.11.006 
Fullerton, G. (2003). When does commitment lead to loyalty? Journal of Service Research, 5(4), 333-344. https://doi.org/10.1177/1094670503005004005

Godin, S. (2009). Purple cow. New York: Penguin Random House.

Gupta, P. \& Harris, J. (2010). How e-WOM recommendations influence product consideration and quality of choice: A motivation to process information perspective. Journal of Business Research, 63(9-19), 1041-1049. https://doi.org/j.jbusres.2009.01.015

Herold, K. (2015). Impact of Word of Mouth on Consumer Making: An Information Processing Perspective in the Context of A High Involvement Service. [Doctoral dissertation]. Lappeenranta University of Technology, Lappeenranta, Finland. Available: http://urn.fi/URN:ISBN:978-952-265-861-6

Jaakkola, E. \& Aarikka, L. (2018). Customer referencing as bussines actor engagement behavior. creating value in and beyond triadic settings. Industrial marketing management, 80, 27-42. https://doi.org/10.1016/j.indmarman.2018.06.014

Karlíček, M., Tomek, I. \& Křížek, M. (2010). Word-of-Mouth Marketing: An Integrated Model. Ekonomika a Management, (3), 1-11. Available: https://www.vse.cz/eam/109

Kim, A. \& Johnson, K. (2016). Power of consumers using social media : examining the influences of brand-related user-generated content on facebook. Computers in human behavior, 58, 98-108. https://doi.org/10.1016/j.chb.2015.12.047

Kotler, P., Kartajaya, H. \& Setiawan, I. (2010). Marketing 3.0: From products to customers to the human spirit. Singapore: John Wiley \& Sons.

Kozinets, R., de Valck, K., Wojnicki, A. \& Wilner, S. (2010). Networked narratives: understanding word-of-mouth marketing on line comunities. Journal of marketing, 74(2), 71-89. https://doi.org/10.1509/jm.74.2.71

Le, T., Dobele, A. \& Robinson, L. (2019). Information sought by prospective students from social media electronic word-of-mouth during the university choice process. Journal of Higher Education Policy and Management, 41(1), 18-34. https://oi.org/10.1080/1 360080X.2018.1538595

Lehmann, W. (2017). The influence of electronic word-of-mouth on college search and choice. College and University, 92(4), 8-11.

Libai, B., Bolton, R., Bügel, M. S., Ruyter, K., Götz, O. \& Stephen, A. T. (2010). Customerto-customer interactions: broadening the scope of word of mouth research. Journal of Service Research, 13(3), 267-282. https://doi.org/10.1177/1094670510375600

López, M. y Sicilia, M. (2013). Boca a boca tradicional vs electrónico. La participación como factor explicativo de la influencia del boca a boca electrónico. Revista Española de Investigaciones de Marketing, 17(1), 7-38. https://doi.org/10.1016/S11381442(14)60017-6

Mazzarol, T., Sweeney, J. C. \& Soutar, G. N. (2007). Conceptualizing word-of mouth activity, triggers and conditions: an exploratory study. European Journal of Marketing, 41(11), 1475-1494. https://doi.org/10.1108/03090560710821260 
Olarte, J. (2020). Factores que predicen la permanencia estudiantil: análisis en un escenario de educación técnica. Cultura, Educación y Sociedad, 11(1), 25-38. https://doi. org/10.17981/cultedusoc.11.1.2020.02

Olle, R. y Riu, D. (2010). El nuevo Brand Management: Lecciones de la indiferenciación de las marcas. Barcelona: Deusto-Planeta de Agostini.

Park, H. \& Kim, Y. (2014). The role of social network websites in the consumer-brand relationship. Journal of retailing and consumer services, 21(4), 460-467. https://doi. org/10.1016/j.jretconser.2014.03.011

Reinares, P. \& Reinares, E. (2007). Analysis of the contents of the advertising saturation on television. Esic Market, 126, 23-94. Available: https://www.esic.edu/documentos/ revistas/esicmk/070118_142648_I.pdf

Salmi, J. (2009). El desafio de crear universidades de clase mundial. Washington, D.C.: Banco Mundial.

Scheers, L. \& Prinsloo, C. (2014). Investigating word of mouth as advertising tool for mobile devices in South Africa. International Journal of Academic Research in Business and Social Sciences, 4(11), 332-354. https://doi.org/10.6007/IJARBSS/V4-I11/1315

Sernovitz, A., Godin, S. y Kawasaki, G. (2009). El marketing de boca en boca. Buenos Aires: Kaplan.

Sham, M. \& Nasr, M. (2016). The effects of a brands online presence on brand awareness and brand loyalty: A study of UAE Millenials. International journal of media, journalism and mass communications, 2(1), 39-50. https://doi.org/10.20431/24549479.0202004

Soares, M., Lapuente, I. y De matos, C. (2015). Recomendación boca a boca incentivada: eso tambien vale? Brazilian Business Review, 96-124. Disponible en http://www. bbronline.com.br/index.php/bbr/issue/archive

Solomon, R. (2013). Comportamiento del consumidor. Naucalpan de Juárez: Pearson.

SPSS IBM (versión 26). Statistical Package for the Social Sciences. [Programa estadístico informático]. Armonk: IBM. Disponible en https://www.ibm.com/support/pages/spssstatistics-220-available-download

Stachowski, C. (2011). Educational marketing: a review and implications for supporting practice in tertiary education. Educational Management Administration \& Leadership, 39(2), 186-204. https://doi.org/10.1177/1741143210390056

Stauss, B. (2000). Using new media for customer interaction: a challenge for relationship marketing. En, T. Hennig-Thurau, \& U. Hansen, Relationship marketing (pp. 233-253). Berlin: Springer.

Trusoy, M., Buclin, R. \& Pauwels, K. (2009). Effects of word of mouth versus traditional marketing : findings from an internet social networking site. Journal of marketing, 73(5), 90-102. https://doi.org/10.1509/jmkg.73.5.90 
Wolny, J. \& Mueller, C. (2013). Analysis of fashion consumers motives to engage in electronic word-of-mouth comunication trough social media platforms. Journal of marketing management, 29(5-6), 562-583. https://doi.org/10.1080/0267257X.2013.778324

Darío José Espinal Ruiz es Profesional en Cultura Física y Deporte. Especialista en Dirección y Gestión Deportiva y Especialista en Teoría y Metodología del Entrenamiento Deportivo. Magister en Administración; Doctor en Administración, Universidad del Valle (Colombia). https://orcid.org/0000-0003-1191-7976

Natali Cruz González es Administradora de Empresa. Licenciada en Educación Básica con énfasis en Ciencias Naturales y Educación Ambiental. Magister en alta dirección de servicios educativos de la Universidad de San Buenaventura (Colombia). https://orcid. org/0000-0003-0914-6236

Luis Enrique David Tenorio es Administrador de Empresas de la Universidad Libre (Colombia). Especialista en Gerencia Financiera del Politécnico Gran Colombiano (Colombia). Magister en Mercadeo de la Universidad de Manizales (Colombia). https://orcid. org/0000-0002-5326-7353 